Editorial

\title{
Global IR: Interventional Radiology in Developing Countries
}

\author{
David S. Shin ${ }^{1} \quad$ Blaine E. Menon ${ }^{2}$ \\ ${ }^{1}$ Department of Radiology, University of Washington, Seattle, \\ Washington, United States \\ 2Department of Interventional Radiology, University of Miami Miller \\ School of Medicine, Miami, Florida, United States
}

J Clin Interv Radiol ISVIR 2019;3:3-6

Interventional radiology (IR) has grown remarkably in the United States over the past few decades. The expansion of minimally invasive image-guided procedures has brought with it a demonstrable improvement in patient outcomes. For example, percutaneous abscess drainage has shown reduced morbidity compared with operative management and become standard of care in many instances. ${ }^{1}$ It can also serve as a temporizing measure in critically ill patients with complex abscesses who are not surgical candidates. ${ }^{2}$ Several cost-effectiveness analyses provide evidence that image-guided techniques can also be more economical than the invasive alternatives, as demonstrated in studies of chest tubes, ${ }^{3}$ abscess drainage, ${ }^{4}$ biopsies, ${ }^{5}$ portosystemic shunting, ${ }^{6}$ central venous access, ${ }^{7}$ and uterine fibroid treatment. ${ }^{8}$ Despite these proven advantages of many IR procedures in high-income nations, the same sort of revolution in image-guided procedures has not occurred in low- and middle-income countries (LMIC).

Global health is not a single disease issue-quite the opposite. It encompasses a variety of pathologies that cross national borders and require international response. With a fundamental set of techniques and tools, such as the Seldinger technique and a pigtail drainage catheter, IR may be able to treat an incredible variety of disease conditions found all over the globe. This article discusses the expansion of IR in LMIC, focusing on current barriers and opportunities for growth.

\section{Establish the Baseline}

One of the greatest challenges to the development of IR in LMIC is a lack of data on existing practice. To the authors' knowledge, no comprehensive databank exists that records basic facts such as what image-guided procedures are offered, how many are performed, who is performing them, and what clinical outcomes and complications are seen after these procedures. Instead, any information gathering is fragmented by hospitals and competing health systems without easy public access. Establishing a comprehensive,

Address for correspondence David S. Shin, MD, Department of

Radiology, University of Washington, 1959 NE Pacific Street, Box 357115 , Seattle, WA 98195, United States (e-mail: dsshin@uw.edu).

standardized approach to collecting pertinent data must be one of the first steps in building IR practice in LMIC. This database would illuminate regional differences in clinical demand and capacity. It would guide investments in health systems to create maximum development for IR-related care.

Several efforts to meet this goal have emerged over the past decade for diagnostic radiology (DR). RAD-AID (rad-aid. org) is a nonprofit public service organization that began in 2008 to improve radiology resources for developing nations. In 2010 it began implementing a formalized assessment called Radiology-Readiness to determine the baseline capacity for radiology services among LMIC. ${ }^{9}$ Radiology-Readiness examines the availability of physical infrastructure, power supply, workforce, antibiotics, biopsies, and laboratory testing. More specific assessments, such as the IR Readiness Assessment Tool, have also been proposed. ${ }^{10}$ This tool collects information on imaging devices, procedural equipment, personal protective equipment, medications for periprocedural care, and affiliated medical services. Though it is yet unclear whether the scope of this tool will be sufficient, it constitutes a great starting point to assess the clinical and infrastructural capacity of a health care system interested in building a new IR service.

As a parallel, the lack of baseline capacity data has also been a barrier for the development of essential surgical services in LMIC. To overcome this obstacle, the Lancet Commission on Global Surgery has partnered with multiple countries around the world to implement National Surgical, Obstetric, and Anesthesia Planning (NSOAP) to better characterize current resources and needs. ${ }^{11} \mathrm{~A}$ key step in this effort was the commission's ability to bring together a diverse group of experts in the field to advise its creation. Its first meeting in 2014 included professors of surgery, anesthesiology, and obstetrics; directors of public health programs and human rights groups; and managers in the World Health Organization (WHO) and World Bank. One opportunity for IR would be to partner with the Lancet Commission and RAD-AID to coordinate efforts for broader procedural development in LMIC.

License terms

$10.1055 / s-0039-1684880$

ISSN 2457-0214. 
Given the often shared infrastructure necessary for surgical, radiologic, and IR services, this collaborative approach would likely be much more efficient than developing separate and redundant assessment tools.

\section{Invest in Human Capital}

If IR is to grow in LMIC, then so must the human capital necessary for that growth. Radiology training in LMIC is often nonexistent or poorly structured. Designated IR training is even less well established. Currently there are several initiatives to fill this gap for DR. The Radiological Society of North America (RSNA), American College of Radiology (ACR), Royal College of Radiologists (RCR), European Society of Radiology (ESR), International Society of Pediatric Radiology (ISPR), and American Society of Radiology Technologists (ASRT) all have international programs that fund radiology education and service in LMIC.

In the past several years, RAD-AID has developed a growing IR service commitment. Among its initiatives, there is a project to compile a comprehensive manual of IR procedures as part of a Global IR Curriculum. ${ }^{12}$ The manual includes a list of supplies and procedural steps for proper execution of dozens of image-guided procedures and can be used as a reference and teaching guide for providers. This represents one example of how to increase the human capital necessary for an IR service on a broader scale. Combined with recorded lectures and live video communication, this manual could help foster IR training to many more sites than would be possible with onsite training alone.

In the United States and other high-income countries, much of the interventional workload is done by radiologists who complete fellowship-level training. LMICs have a very different workforce at their disposal, often limited to health care providers who practice as generalists. To overcome this difference, many global health organizations use a process called task shifting. Task shifting is defined by the WHO as the movement of specific tasks from "highly qualified health workers to health workers with shorter training and fewer qualifications in order to make more efficient use of the available human resources for health."13 This practice has evolved out of necessity in many low-resource settings. For example, one study of task shifting in sub-Saharan Africa found that nonphysician clinicians (NPCs) performed diagnostic and treatment functions traditionally in the domain of physicians in 25 of the 47 countries investigated. ${ }^{14}$ Twelve of these nations reported NPC performing unspecified minor surgeries in regions without access to fully trained surgeons. In another example, investigators found that general medical officers without formal surgical education were performing cesarean sections, tubal ligations, wound suturing, incision and drainage, hernia repair, and laparotomies in many public district hospitals in Uganda. ${ }^{15}$

Task shifting can expand access to services where there is a lack of fully credentialed providers. IR may be even more amenable to task shifting than surgical specialties because many of the basic IR techniques can be readily learned by motivated providers already versed in anatomy and pathology. In fact, this is already a common practice in the United States with employment of midlevel providers for venous access, fluid collection drainage, centesis, and gastrostomy tube placement. A retrospective review found that in central venous access procedures, practitioner assistants demonstrated no higher complication rates compared with IR physicians. ${ }^{16}$ The same benefits driving this trend in the United Stares-increased access to IR procedures at a lower cost-would no doubt be desirable in LMIC.

\section{Spend Resources Wisely}

One of the most significant barriers to the expansion of IR in LMIC is the cost of DR imaging equipment and facilities. The capital cost of obtaining the machines for imaging can be daunting. A quick search finds that CT scanners can range from around $\$ 65,000$ for refurbished, low-end models to $\$ 2.5$ million for newer models. Magnetic resonance imaging (MRI) scanners are even more costly, ranging up to $\$ 3$ million for a new machine. In a large group of hospitals in the United States, the mean operating costs and charges per procedure of various imaging modalities were reported as follows: computed tomography (CT): \$51 and \$1,565; X-ray and ultrasound (US): \$55 and \$410; nuclear medicine: \$135 and $\$ 1,138$; and MRI: $\$ 165$ and $\$ 2,048 .{ }^{17}$ The capital and operating expenses can pose a large obstacle to initial investment in image-guided procedures in resource-limited settings.

However, it is worth noting that many IR procedures do not require an expensive arsenal of diagnostic scanners. C-arms used for fluoroscopy are much cheaper than CT and MRI scanners, ranging from about $\$ 20,000$ to $\$ 80,000$. Likewise, US units can range from about $\$ 10,000$ up to $\$ 200,000$. Most IR procedures can be done with a combination of US and fluoroscopy guidance. This was demonstrated aboard the medical relief ship Mercy after devastating tsunamis and earthquakes shook regions in South Asia in 2005. Approximately 300 IR procedures were completed on the ship, and over two-thirds of these were performed with fluoroscopy and/or US, including venous access, biliary and abscess drainage, and inferior vena cava (IVC) filter placement, among many others. ${ }^{18}$

Combat settings also provide a relevant framework for IR in LMIC. Portable, rechargeable US units have been used directly in field environments and tent hospitals by the military to perform a vast array of percutaneous procedures such as removal of foreign bodies following a blast injury, placement of drains for fluid collections and abscesses, and placement of both arterial and central venous lines. ${ }^{19}$ For more advanced procedures, high-quality fluoroscopy has also become available in mobile field hospitals and been used for a variety of procedures such as stent graft placement and embolization following traumatic bleeding. ${ }^{19}$ Despite logistical challenges, IR has adapted to deliver high-quality, minimally invasive care in even the most extreme settings. These examples offer invaluable guidance for the development of IR service in LMIC. Many IR procedures can be performed with lower-cost modalities and may even be more costeffective than building a modern DR service in LMIC. 


\section{Create Responsible Interventions}

International aid to improve the health of impoverished nations can take on many forms. A growing trend among health care students and providers is to travel to an underserved region for a short time to provide services that communities may otherwise lack, often called medical service trips (MSTs). A recent ethical discussion explored many of the risks of MST, including: disrespect for misunderstood cultural expectations and values around health; patient harm from substandard care when providers practice beyond their scope of training or without equipment and supplies they require; competition with scarce local health care resources and a dependence on aid from outside the community; and the use of limited local resources for objectives that are defined by the volunteers and not the priorities of the community. ${ }^{20}$ Any effort to develop IR in LMIC must carefully consider how to minimize such transitory relief efforts and focus on building a sustainable practice.

In this regard, several organizations have released best practice guidelines to which health care providers and educators can refer. For example, the Sphere Project has pioneered a set of minimum standards in humanitarian assistance for over 20 years. MSF has a wealth of guidelines on international assistance ranging from technical advice on how to approach specific diseases to broader education of sociolegal considerations pertinent to global health. Perspectives from the experiences of groups such as the Children's Health International Medical Project of Seattle describe core principles that similar groups can use when planning their own efforts. ${ }^{21}$ Efforts to expand IR must not only focus on improving care but must also build an ethical, respectful, and responsible practice.

\section{Conclusion}

Interventional radiology is sometimes seen as an "elite" subspecialty heavily dependent on technological advancements. At first glance, this may seem like an obvious barrier to expanding IR in low-resource settings. However, IR is also a field founded upon innovation, creativity, and vision to find novel, minimally invasive ways to treat patients safely and effectively. With this in mind, IR has much to offer global health. Many procedures can be done with relatively inexpensive imaging modalities and can be taught to midlevel providers to increase access without sacrificing safety. The misconception that IR belongs only to rich nations must be replaced by a creativity to find solutions to bring IR to developing countries.

\section{Conflict of Interest}

The authors declare that there is no conflict of interest regarding the publication of this article.
2 vanSonnenberg E, Wing VW, Casola G, et al. Temporizing effect of percutaneous drainage of complicated abscesses in critically ill patients. AJR Am J Roentgenol 1984;142(4):821-826

3 LaRoy JR, White SB, Jayakrishnan T, et al. Cost and morbidity analysis of chest port insertion: interventional radiology suite versus operating room. J Am Coll Radiol 2015;12(6):563-571

4 Wright TN, Gilligan L, Zhurbich O, Davenport DL, Draus JM Jr. Minimally invasive drainage of subcutaneous abscesses reduces hospital cost and length of stay. South Med J 2013;106(12):689-692

5 Pasha T, Gabriel S, Therneau T, Dickson ER, Lindor KD. Cost-effectiveness of ultrasound-guided liver biopsy. Hepatology 1998;27(5):1220-1226

6 D'Amico G, Luca A. TIPS is a cost effective alternative to surgical shunt as a rescue therapy for prevention of recurrent bleeding from esophageal varices. J Hepatol 2008;48(3):387-390

7 Noh HM, Kaufman JA, Rhea JT, Kim SY, Geller SC, Waltman AC. Cost comparison of radiologic versus surgical placement of long-term hemodialysis catheters. AJR Am J Roentgenol 1999;172(3):673-675

8 Jun F, Yamin L, Xinli X, et al. Uterine artery embolization versus surgery for symptomatic uterine fibroids: a randomized controlled trial and a meta-analysis of the literature. Arch Gynecol Obstet 2012;285(5):1407-1413

9 RAD-AID International. Radiology-Readiness: A Framework for Implementing in Resource-Limited Regions [Internet]. Chevy Chase, MD: RAD-AID International; c2008-2017. https:// www.rad-aid.org/resource- center/radiology-readiness/

10 Kline AD, Dixon RG, Brown MK, Culp MP. Interventional Radiology Readiness Assessment Tool for global health. J Glob Radiol 2017;3(1):2

11 Meara JG, Hagander L, Leather AJM. Surgery and global health: a Lancet Commission. Lancet 2014;383(9911):12-13

12 RAD-AID Interventional Radiology. (2018). Retrieved from https://www.rad-aid.org/programs/interventional-radiology/

13 World Health Organization, PEPFAR \& UNAIDS. Task shifting: rational redistribution of tasks among health workforce teams: global recommendations and guidelines. Geneva: World Health Organization. 2007. Available at: http://www. who.int/iris/handle/10665/43821

14 Mullan F, Frehywot S. Non-physician clinicians in 47 sub-Saharan African countries. Lancet 2007;370(9605): 2158-2163

15 Ozgediz D, Galukande M, Mabweijano J, et al. The neglect of the global surgical workforce: experience and evidence from Uganda. World J Surg 2008;32(6):1208-1215

16 Benham JR, Culp WC, Wright LB, McCowan TC. Complication rate of venous access procedures performed by a radiology practitioner assistant compared with interventional radiology physicians and supervised trainees. J Vasc Interv Radiol 2007;18(8):1001-1004

17 Sistrom CL, McKay NL. Costs, charges, and revenues for hospital diagnostic imaging procedures: differences by modality and hospital characteristics. J Am Coll Radiol 2005;2(6):511-519

18 Ferrara S. Interventional radiology in the austere environment. Semin Intervent Radiol 2010;27(1):3-13

19 Mendoza J, et al. Interventional radiology in the combat environment. Curr Trauma Rep 2017;3(3):249-256

20 Stone GS, Olson KR. The ethics of medical volunteerism. Med Clin North Am 2016;100(2):237-246

21 Suchdev P, Ahrens K, Click E, Macklin L, Evangelista D, Graham E. A model for sustainable short-term international medical trips. Ambul Pediatr 2007;7(4):317-320

\section{References}

1 vanSonnenberg E, Wittich GR, Goodacre BW, Casola G, D’Agostino HB. Percutaneous abscess drainage: update. World J Surg 2001;25(3):362-369, discussion 370-372 
